\title{
Application of High-Voltage Discharges for Disinfecting Water
}

\author{
Alexander A. Belov, Alexey N. Vasilyev \\ Federal Scientific Agroengineering Center VIM, Russia
}

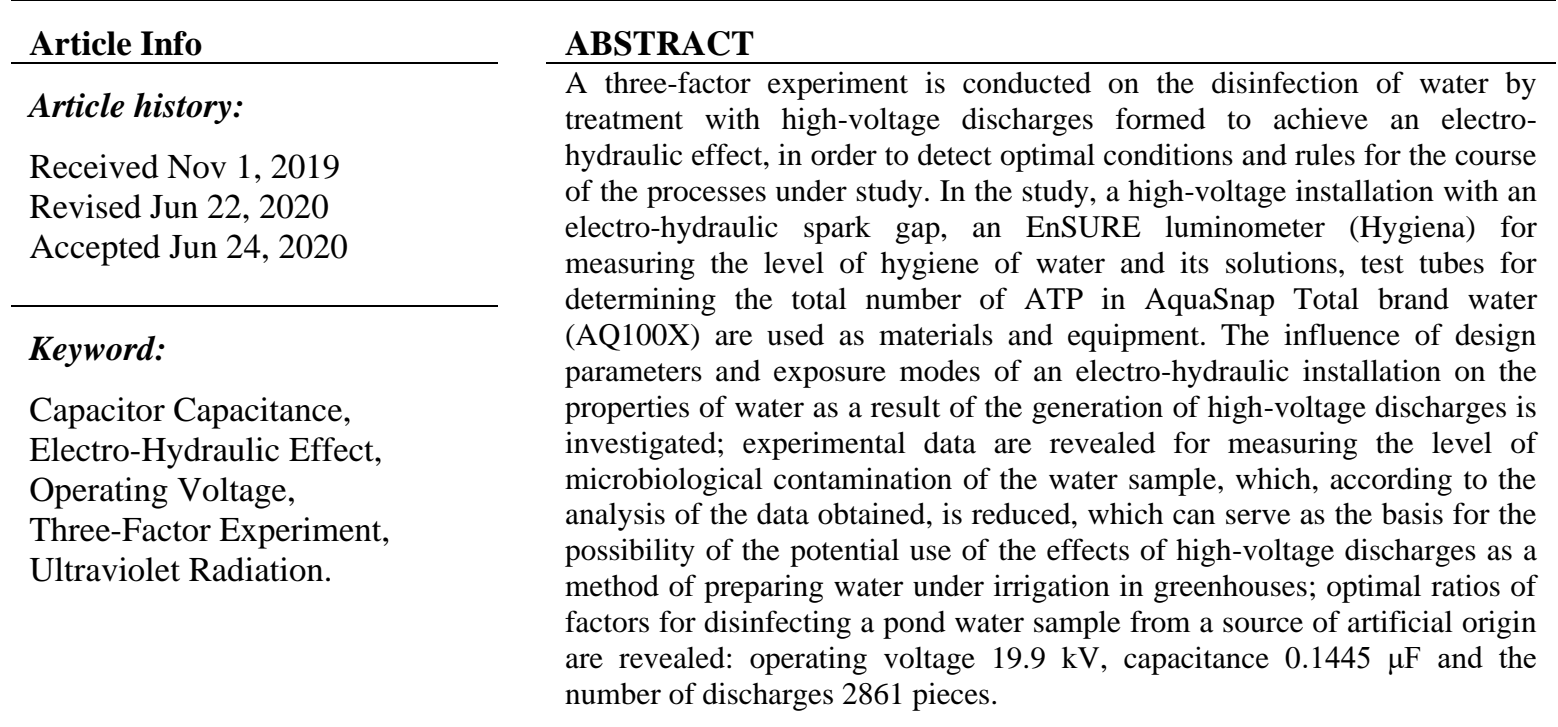

Copyright $\odot 2020$ Institute of Advanced Engineering and Science. All rights reserved.

\section{Corresponding Author:}

Alexander A. Belov,

Federal Scientific Agroengineering Center VIM,

109428, 1st Institute Passage, 5, Moscow, Russia.

Email: belalexan85@gmail.com

\section{INTRODUCTION}

Prevention of diseases of plants grown in greenhouse systems is no less significant for crop productivity than building and maintaining a balanced chemical composition and content of macro- and microelements in nutrient solution [1]. The use of drainage water, water from rivers and open reservoirs for irrigation of plants without the use of sterilization and disinfection means can lead to infection and the spread of infection in leaves, stems and fruits. Nutrient solutions for irrigation of greenhouse crops, as such, are a favorable environment for the development of microorganisms that provoke various diseases of greenhouse plants. Plant diseases can be bacterial, which are most often manifested by putrefactive diseases, and viral, the evidence of which is the mosaic contour, depending on the source of infection. In addition to the above, diseases of a non-parasitic nature should be noted [2]. Through the nutrient solution during irrigation, the keel, black leg, white rot, gall nematode, strick, late blight and other diseases can be transmitted. Whatever the cause of infection, the disease worsens the growth and development of the greenhouse crop, resulting in a decrease in fruiting. The result may be the death of the plant.

Modern greenhouses in the preparation of nutrient solutions use various methods for disinfecting water [3]. A chemical method for controlling bacterial and viral morbidity can be used. However, the introduction of biocidal and antiseptic substances into the nutrient solution can lead to a decrease in the environmental quality of the product. Alternative and more environmentally friendly is the use of water ozonation technology. Ozone is able to inactivate microorganisms in water, but at high temperatures and acidity, ozone requires more time for exposure due to its rapid decay [4]. A high concentration of ozone in water can have a negative effect on the root system of plants. Water treatment by ultraviolet radiation most effectively destroys bacterial microflora [5]. However, in the presence of mechanical impurities in water, the quality of disinfection is reduced; the technology requires the installation of additional filters for cleaning 
nutrient solutions. The cost of ultraviolet water treatment, including the cost of energy, installation and maintenance, is relatively high [6-7]. There are developments on water purification by exposure to solar radiation [8]. In this regard, it is urgent to develop an environmentally friendly method of water disinfection for the destruction of bacterial microflora in water under the conditions of water treatment of nutrient solutions for irrigation. It is proposed to use electro-hydraulic (EH) treatment of water with high-voltage discharges as a disinfection in the preparation of nutrient solutions. According to literature sources, the electro-hydraulic effect is a powerful source of ultrasound, as well as ultraviolet and X-ray radiation, capable of forming atomic oxygen. All this can destroy pathogenic microflora in water [9]. Scientists have also studied the cleaning bactericidal effect of treating water with high-voltage electric discharges, and a number of works on this topic have been published [10].

However, the methods and means of measurement used in this case are not relevant, and do not allow dynamic observations, which is important when studying real processes of changing the physical, chemical, and microbiological properties of an object. The properties of the phenomenon of water disinfection should be investigated in the search for a rational sequence of data acquisition, so that with minimized time and financial costs produce the most reliable information about the object of interest. To find the optimal conditions and rules for the flow of the studied processes occurring during electro-hydraulic disinfection of water and its solutions, it is necessary to use the full factorial experiment as a method of planning the experiment. Therefore, the aim of this article is to study the possibility of using high-voltage discharges for disinfecting water as a result of the experiment.

\section{RESEARCH METHOD}

When conducting research in this article, the following equipment and materials were used:

- high-voltage installation with an EH-arrester;

- EnSURE luminometer (Hygiena) for measuring the level of hygiene of water and its solutions;

- test tubes for determining the total number of ATP in water brand AquaSnap Total (AQ100X).

The applied measurement methods are presented in the following form:

- multifactorial planning method;

- Appendix to the certificate No. 54301 on type approval of measuring instruments; measurement technique with an EnSURE luminometer (Hygiena).

The design parameters and the technological mode of operation of a high-voltage installation with an EH discharger are as follows:

- operating voltage supplied to the electrodes $14-40 \mathrm{kV}$ (measured by a multimeter indirect method on a divider step-up transformer);

- a capacitor battery of $0.075-0.2 \mu \mathrm{F}$ (parallel connection of four capacitors with a capacity of 0.025 $\mu \mathrm{F})$

- additional spark gap of 2-8 $\mathrm{mm}$ (the distance between the discharge aluminum balls);

- working gap of 2.5-8 mm (distance between electrodes)

- electrode system - tip-disc (disc diameter $66 \mathrm{~mm}$ );

- frequency of spark electric discharges $3 \mathrm{~Hz}$;

- the number of discharges $1000-4000$ pcs.

To measure the hygiene level of water and its solutions, an EnSURE luminometer (Hygiena) was used. The device works in conjunction with test tubes to determine the total number of ATP (adenosine triphosphate) in AquaSnap Total (AQ100X) brand water.

Currently, there is a modern, fast, and at the same time accurate method for assessing the degree of microbiological safety of water and its solutions. A method based on the luminometric determination of the amount of intracellular ATP (adenosine triphosphate). The principle of operation of the aforementioned luminometer is to determine the level of adenosine triphosphate (ATP) - a universal energy molecule found in all plant, animal and bacterial cells, including yeast and mold. The value of ATP directly depends on the degree of microbial contamination and organic pollution. Thus, the concentration of ATP reflects the value of the total microbial number, which means it indicates the level of hygiene.

ATP level is measured in relative light units - RLU. One RLU unit corresponds to 1 femtomolecules (femtomol.) (10-15 mol.) ATP. Such an amount of intracellular ATP is contained in several microbial cells, which is equivalent to single CFU on a nutrient medium. The luminometer is based on the principle of bioluminescence and refers to screening methods that allow you to quickly and safely identify potentially dangerous biological risks.

The grounds for choosing a technical measuring instrument, more precisely - a luminometer:

1. The operating measurement range of 0-9999 RLU satisfies the expected conditions of the experiment on EH-exposure in terms of the interval of changes in microbiological contamination of water 015 RLU. 
2. Speed (obtaining measurement results within a few seconds), ease of use (portability), ease of maintenance (auto-calibration), high sensitivity (10-15 ATP molecules $=1$ RLU).

3 . The advantage of this device in comparison with microbiological analyzes is that it allows you to determine the presence of contaminants, both inorganic and organic, that is, animal and plant origin, which contribute to the growth and reproduction of bacteria.

The complete factorial experiment is performed according to the optimization algorithm by implementing the following operations: selecting the regression equation, drawing up a plan for the full factorial experiment, calculating the regression coefficients, estimating the significance of these coefficients, analyzing the regression equation, and searching for the optimum [11-12].

\section{RESULTS AND DISCUSSION}

The generation of high voltage discharges is ensured by the developed high voltage installation. The main prerequisites that provide a variety of technological capabilities of the electro-hydraulic effect are methods for increasing the length of discharges. The first method is to obtain discharges in conducting media that have an ionizing property when an electric current flows through them, by reducing the working area of the positive electrode while simultaneously increasing the working area of the negative electrode. The electrodes to which a constant voltage is applied, the amplitude of which reaches several tens of kilovolts, are therefore the high-voltage and main process-forming parts of the electro-hydraulic device. The electrohydraulic effect is achieved in the device due to high hydraulic impulse pressures, mechanical collisions of water against the device walls, portioned light, thermal, ultraviolet radiations, as well as due to repeated ionization processes of compounds and elements contained in the working aqueous medium.

The working area of the positive electrode is the surface that is in direct contact with the working medium, which is water. The working area of the negative electrode is considered to be a surface that also has contact with water, but which has some distinctive structural and functional features.

The positive electrode is a metal stud screwed into the insulator by thread. The material of the insulator can be a fluoroplastic or caprolon with a high electric strength, not less than $25 \mathrm{kV} / \mathrm{mm}$. A high level of current insulation of the dielectric material is required to eliminate breakdown on the metal parts of the device. The threaded connection of the stud and insulator is necessary, on the one hand, for a reliable and tight fit and hold of the stud relative to the insulator. On the other hand, the design is made taking into account the possibility of its movement in the insulator. This property is due to the fact that as the operating time during electro-hydraulic shocks, the working surface of the positive electrode-stud wear down, grinds off and the working area of the electrode burns out the insulator and is drowns in it. Therefore, the hairpin needs to be made adjustable in terms of reach relative to the ends of the insulating material, whether it be caprolon or fluoroplastic.

The negative electrode is an assembly of stud and disc. The disk is a tip, nozzle, or a kind of plane that receives discharges from the positive electrode, that is, it carries the main load with a high voltage load. In addition, the negative electrode must be connected to ground. By discharges we mean pulsed electric spark and corona discharges formed due to the flow of electric current.

In order to enhance the effect of electro-hydraulic shocks, conditions are created for the most efficient conversion of electrical energy into mechanical energy. At the same time, it is understood that the discharge sparks formed between the electrodes are a means, organ, or instrument of transformation and transfer of energy to an aqueous medium.

The authors deduced the scientific assumption that the increase in the length of spark electric discharges is possible by method, which consists in generating discharges not on a flat, but on a hemispherical disk. The device was simulated in a graphical editor to find out the types of generated modes in accordance with figure 1 (a). Thus, changes are made to the design of the negative electrode in accordance with figure 1 (b).

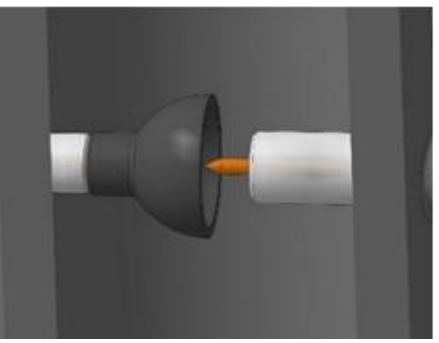

a)

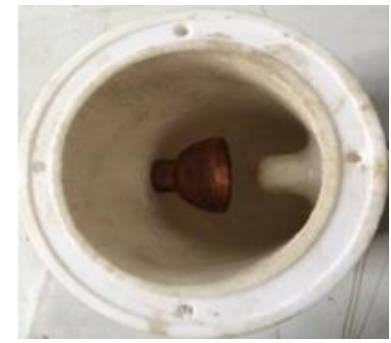

b)

Figure 1. Electrodes of an electro-hydraulic device: a) graphical model; 
b) physical model

The improvement of the electro-hydraulic process is based on several principles of the theory of electromagnetic waves. The ideas of controlled addition and directed action of electromagnetic waves made it possible to argue that the process of generating spark discharges will be simplified by using a tip in the shape of a hemisphere. The maximum effect from exposure to electromagnetic waves will be with a higher quality factor of the hemisphere material. The application of the developed electrode will allow you to choose the minimum capacitance of the capacitors for the accumulation and subsequent discharge of it into the discharge region. The voltage that is supplied to the electrodes will also be lower compared to the analog, disk-shaped tip. To ensure the result of electro-hydraulic processing, fewer discharges will be required, and therefore a shorter duration of operation of the device. This will increase the productivity of the process.

The electro-hydraulic effect is applied in various fields of the national economy quite limitedly. This is facilitated by the required compliance with safety regulations. However, when using the proposed technical solutions, the high-voltage technology for processing raw materials is slightly simplified. In light of the foregoing, it is possible to expand the scope of the electro-hydraulic effect, including in agriculture for disinfecting water, since the critical conditions for voltages, capacities and time of the electro-hydraulic process will be milder and more acceptable.

\section{The choice of the regression equation.}

According to a sifting experiment carried out according to the Plackett-Berman method and the random balance method, the significant factors influencing the process of electro-hydraulic treatment of water and its solutions are the magnitude of the applied (working) voltage to the electrodes of the EH installation, the battery capacity of the storage capacitors and the number of generated pulses or electric discharges [13]. In this regard, the number of factors adequately determining the process is three. In general form, the regression equation without members of higher orders is written as follows:

$$
y=b_{0}+b_{1} \cdot x_{1}+b_{2} \cdot x_{2}+b_{3} \cdot x_{3}+b_{1,2} \cdot x_{1} \cdot x_{2}+b_{1,3} \cdot x_{1} \cdot x_{3}+b_{2,3} \cdot x_{2} \cdot x_{3}+b_{1,2,3} \cdot x_{1} \cdot x_{2} .
$$

where $x_{1}, x_{2}, x_{3}$ - the values of the factors; $b_{0}$ - free term equal to the output at $x_{i}=0 ; b_{1}, b_{2}, b_{3}-$ regression coefficients of the corresponding factors that determine the influence of a factor on the studied process; $b_{1,2}, b_{1,3}, b_{2,3}$ - regression coefficients for the products of factors that indicate the presence of a double interaction between factors; $b_{1,2,3}$ - regression coefficient indicating a triple interaction of factors.

\section{Planning a multifactorial experiment.}

Based on preliminary experiments, taking into account theoretical considerations, a conditional zero level $O_{x i}$ is chosen for all the factors studied, that is, such values of variables within which the process study will debut within the boundary frames in order to identify the direction of change of the selected conditional zero level to the optimal values of the factors.

For the operating voltage factor, $18.5 \mathrm{kV}$ is taken as the conditional zero level. For the capacitance factor of a capacitor bank, $0.1375 \mu \mathrm{F}$ is taken as the conditional zero level. For the factor of the number of discharges, 2500 pieces are taken as the conditional zero level.

For the above factors, units of variation of $\lambda \mathrm{i}$ are selected based on preliminary experimental data and relying on intuition. This refers to the values by which the conditions for each factor vary in ascending and descending order from the conditional zero level in a specific alternate repetition of experience.

The units of variation of the three previously selected factors are defined by the following:

1) the lower and upper levels of operating voltage - 14 and $23 \mathrm{kV}$, respectively;

2) the lower and upper capacitance levels of the capacitors are 0.075 and $0.2 \mu \mathrm{F}$, respectively;

3 ) the lower and upper levels of the number of discharges - 1000 and 4000 pcs. respectively.

The design parameters of the EH installation, namely, the working and forming gaps, were selected empirically, depending on the ratio of the levels of operating voltage and capacitance of the capacitors.

The accuracy of operating voltage levels up to tenths, which is provided by the master control and measuring multimeter, is determined by the desire to provide the same energy in each experiment. The minimum operating voltage corresponding to the lower level is set from the standpoint of achieving the result of the electro-hydraulic effect. So at a lower voltage, taking into account the connection of the maximum capacitor capacitors, the EH effect is not observed.

Capacitor bank capacitance levels are selected depending on preliminary experiments and the results of studies of a high-voltage discharge in water by other authors [14-15].

The levels $O_{x i}-\lambda_{i}$ and $O_{x i}+\lambda_{i}$ are denoted by -1 and +1 . Perhaps the representation in a simpler notation "-" and "+", respectively. This designation is considered coded. It turns out that when choosing $O_{x i}$ and $\lambda_{i}$ in fact, for each value of all the selected factors, it is possible to express them in encoded form. 
Thus, the conditional zero level and units of variation of the design of the full three-factor experiment in arbitrary units are as follows according to table 1.

Table 1. Conditional zero level and units of variation plan full three-factor experiment

\begin{tabular}{|c|c|c|c|c|c|}
\hline № & Investigated factor & $O_{x i}$ & $\lambda_{i}$ & -1 & +1 \\
\hline 1 & $x_{1}$ - operating voltage & 18,5 & 4,5 & 14 & 23 \\
\hline 2 & $x_{2}$ - capacitor capacitance & 0,1375 & 0,0625 & 0,075 & 0,2 \\
\hline 3 & $x_{3}-$ number of discharge & 2500 & 1500 & 1000 & 4000 \\
\hline
\end{tabular}

The units of variation are selected in order to provide a constant limiting amount of energy that accumulates in the capacitors of a high-voltage installation and is generated in the discharge gap of an electro-hydraulic device. Thus, $20 \mathrm{~J}$ of an averaged energy value is supplied to the working spark gap in each experiment.

The level of microbiological contamination of a water sample was measured with an EnSURE (Hygiena) luminometer. The output, that is, the level of disinfection, must be considered specific relative to the volume of the water sample. In this experiment, a one-time loading was carried out on a volume of water of 2.5 liters. The water source adopted a sample or sample of pond water from a source of artificial origin, which serves for agrotechnological purposes for irrigation and irrigation in crop production or for the evaporation of farm animals. Experimental studies were carried out in the laboratory at room temperature 25 ${ }^{\circ} \mathrm{C}$. According to the measurement procedure, 32 water samples were formed, each of which was a volume of $120 \mathrm{ml}$. The control value of the level of microbiological contamination of the water sample was 1208 RLU, as an average of three options for repetition over the entire volume of water in the source in accordance with figure 2(a). The results of measurements with a luminometer obtained during studies of changes in the microbiological composition of water under certain conditions are presented in accordance with figure 2(b, c).

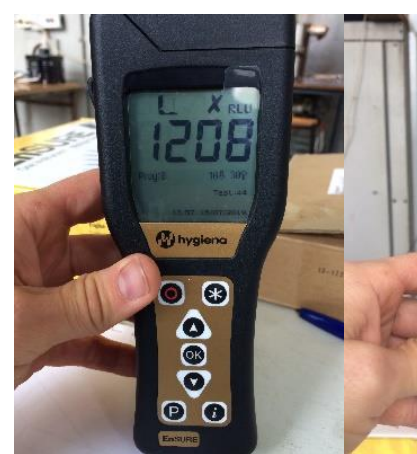

a)

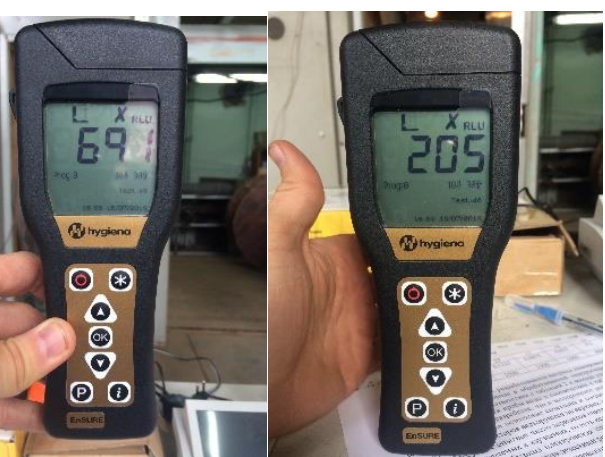

c)

Figure 2. The results of measurements with a luminometer obtained during studies of changes in the microbiological composition of water: a) a control sample of water without treatment; b) a test sample of water after $\mathrm{EH}$ treatment at an operating voltage of $14 \mathrm{kV}$, capacitors $0.075 \mu \mathrm{F}$, and the number of discharges 1000 pieces; c) a test sample of water after $\mathrm{EH}$ treatment at an operating voltage of $14 \mathrm{kV}$, capacitance of 0.2 $\mu \mathrm{F}$, and the number of discharges $4000 \mathrm{pcs}$

Following the choice of $O_{x i}$ and $\lambda_{i}$, a planning matrix for the output of the level of microbiological contamination of the water sample is developed in accordance with table 2 . It should be taken into account during their development that all possible combinations and connections of the values of the factors should be exhaustively worked out in the experiment. These values of the factors vary at the upper and lower levels $\left(O_{x i}-\lambda_{i}, O_{x i}+\lambda_{i}\right)$. The required experimental number of options is considered equal to $2^{i}=N$, where $\mathrm{i}$ is the number of tested and studied, that is, investigated factors.

Table 2. Matrix of planning the level of microbiological contamination of water samples

\begin{tabular}{|c|c|c|c|c|c|c|c|c|c|c|c|c|}
\hline \multirow{2}{*}{$\begin{array}{c}\text { Number of } \\
\text { option }\end{array}$} & \multicolumn{4}{|c|}{ Planning } & \multicolumn{4}{|c|}{ Calculation } & \multicolumn{4}{|c|}{ Output } \\
\hline & $x_{0}$ & $x_{1}$ & $x_{2}$ & $x_{3}$ & $x_{1} \cdot x_{2}$ & $x_{1} \cdot x_{3}$ & $x_{2} \cdot x_{3}$ & $x_{1} \cdot x_{2} \cdot x_{3}$ & $y_{N}^{l}$ & $y_{N}^{2}$ & $y_{N}{ }^{3}$ & $y_{N}$ \\
\hline 1 & + & - & - & - & + & + & + & - & 693 & 698 & 682 & 691 \\
\hline 2 & + & + & - & - & - & - & + & + & 283 & 293 & 291 & 289 \\
\hline 3 & + & - & + & - & - & - & - & + & 564 & 560 & 577 & 567 \\
\hline 4 & + & + & + & - & + & + & - & - & 88 & 87 & 80 & 85 \\
\hline 5 & + & - & - & + & + & + & - & + & 307 & 305 & 294 & 302 \\
\hline
\end{tabular}




\begin{tabular}{cccccccccccccc}
\hline 6 & + & + & - & + & - & - & - & - & 89 & 92 & 86 & 89 \\
7 & + & - & + & + & - & - & + & - & 207 & 200 & 208 & 205 \\
8 & + & + & + & + & + & + & + & + & 69 & 74 & 76 & 73 \\
\hline
\end{tabular}

Experimental data revealed a decrease in the level of microbiological contamination of water samples, which is shown in columns 10-13 of the table of the planning matrix. An analysis of the results of the study of changes in the microbiological composition of water and its solutions after $\mathrm{EH}$ treatment revealed a tendency to decrease to varying degrees, depending on technological conditions.

Factors that play an important role in the inactivation of microorganisms in water during spark generation are the generation of strong ultraviolet radiation, a strong electric field, free radicals, shock waves and metal nanoparticles resulting from the destruction of electrodes. The energy is stored in the plasma in the form of ionization, excitation and kinetic energy of the random movement of particles. The accumulated energy is removed from the plasma by electromagnetic radiation, shock waves and heat transfer to neighboring water molecules, since the spark channel emits light and expands in size. Depending on the type of discharge and the total energy input, about thirty percent of the plasma energy can be emitted in the form of ultraviolet radiation.

It was found that the temperature of water samples increased upon treatment with EH discharges in the amount of 1000 from $25^{\circ} \mathrm{C}$ to $40{ }^{\circ} \mathrm{C}$ and when exposed to $\mathrm{EH}$ discharges in the amount of 4000 from 25 ${ }^{\circ} \mathrm{C}$ to $48{ }^{\circ} \mathrm{C}$. Temperature rises due to heat transfer from spark discharges to water samples. Changing the temperature of water samples does not affect the disinfection process.

The second column of the planning matrix includes the value of the dummy variable $x_{0}=+1$, the introduction of which is conditional on compliance with the formalities in the calculation conditions $b_{0}$. The planning of the experiment consists of the third, fourth and fifth columns, in which the values of the variables $x_{1}, x_{2}, x_{3}$ are entered. The sixth, seventh, eighth and ninth columns are calculated, containing the values of the products $x_{1} \cdot x_{2}, x_{1} \cdot x_{3}, x_{2} \cdot x_{3}, x_{1} \cdot x_{2} \cdot x_{3}$, which are entered into the planning matrix in order to further determine the regression coefficients $b_{1,2}, b_{1,3}, b_{2,3}, b_{1,2,3}$. The tenth, eleventh and twelfth contain the values of the output, that is, the results of measurements and observations in each of the 8 experiments. In the first row of the planning matrix corresponding to the first version of the experiment, all three variables are located at the lower level. In the second row of the planning matrix corresponding to the second variant of the experiment, the first variable is placed at the upper level, the second and third variables are placed at the lower level, and so on.

\section{Calculation of regression coefficients.}

The matrix for planning the level of microbiological contamination of a water sample is solved by determining the regression coefficients according to well-known formulas. Thus, it turns out:

$$
\begin{gathered}
y=287.6-153.6 \cdot x_{1}-55.1 \cdot x_{2}-120.4 \cdot x_{3}+0.1 \cdot x_{1} \cdot x_{2}+67.4 \cdot x_{1} \cdot x_{3}+26.9 \cdot x_{2} \cdot x_{3}- \\
20.1 \cdot x_{1} \cdot x_{2} \cdot x_{3},
\end{gathered}
$$

Assessment of the significance of regression coefficients thus are determined:

- line dispersion $S^{2}\left[y_{N}^{k}\right]=67 ; 28 ; 79 ; 19 ; 49 ; 9 ; 19 ; 13$;

- reproducibility dispersion $S^{2}[y]=35.375$;

- mean variance $S^{2}[\bar{y}]=11.79$;

- regression coefficient variance $S^{2}\left[b_{i}\right]=1.47$

From the variance of the regression coefficients, the error of the regression coefficients is determined $S\left[b_{i}\right]=1.21$. The number of degrees of freedom 16. The distribution of the student criterion is 2.92 with $99 \%$ confidence. It is further stated that for a confidence level of 0.99 , the coefficients $b_{1}, b_{2}, b_{3}$, $b_{1,2}$ are significant.

\section{Analysis of the regression equation.}

In this regard, it is argued that the level of disinfection of the water sample is influenced by all selected factors, such as operating voltage, capacitance of capacitors and the number of discharges. In addition, it is noted that the pair interaction of the first and second factors affects the process.

Testing the possibility of describing the disinfection process of a water sample by a linear model is carried out according to the Fisher criterion. The output values are determined by the regression equation with discarded members of pair interactions for the values of each factor in accordance with the option. The variance of the model inadequacy without pair interactions is calculated by comparing it with the variance of reproducibility. It turns out the estimated value of the estimation of variances is less than the table. Therefore, the output in this problem is sufficiently described by the linear regression equation without pairwise interactions.

Search for the optimum. 
Optimization of factors and output is carried out according to the steep climb program [16]. According to previous calculations $b_{1} \cdot \lambda_{1}=691.2 ; b_{2} \cdot \lambda_{2}=3.44 ; b_{3} \cdot \lambda_{3}=180600$. In accordance with Table 3, the steep climb plan in the coded designation is as follows.

\begin{tabular}{ccccccc}
\multicolumn{7}{c}{ Table 3. Conditions in coded notation } \\
\hline $\begin{array}{c}\text { Number } \\
\text { of option }\end{array}$ & 1 & 2 & 3 & 4 & 5 & 6 \\
\hline$x_{1}$ & $0_{x 1}$ & $0+0.001 \cdot b_{1} \cdot \lambda_{1}$ & $0+0.002 \cdot b_{1} \cdot \lambda_{1}$ & $0+0.003 \cdot b_{1} \cdot \lambda_{1}$ & $0+0.004 \cdot b_{1} \cdot \lambda_{1}$ & $0+0.005 \cdot b_{1} \cdot \lambda_{1}$ \\
$x_{2}$ & $0_{x 2}$ & $0+0.001 \cdot b_{2} \cdot \lambda_{2}$ & $0+0.002 \cdot b_{2} \cdot \lambda_{2}$ & $0+0.003 \cdot b_{2} \cdot \lambda_{2}$ & $0+0.004 \cdot b_{2} \cdot \lambda_{2}$ & $0+0.005 \cdot b_{2} \cdot \lambda_{2}$ \\
$x_{3}$ & $O_{x 3}$ & $0+0.001 \cdot b_{3} \cdot \lambda_{3}$ & $0+0.002 \cdot b_{3} \cdot \lambda_{3}$ & $0+0.003 \cdot b_{3} \cdot \lambda_{3}$ & $0+0.004 \cdot b_{3} \cdot \lambda_{3}$ & $0+0.005 \cdot b_{3} \cdot \lambda_{3}$ \\
\hline
\end{tabular}

Next, the conditions in the coded designation are replaced by the conditions in real terms in accordance with table 4 .

Table 4. Conditions in real terms

\begin{tabular}{ccccccc}
\hline $\begin{array}{c}\text { Number of } \\
\text { option }\end{array}$ & 1 & 2 & 3 & 4 & 5 & 21.9 \\
\hline$x_{1}$ & 18.5 & 19.2 & 19.9 & 20.6 & 21.3 & 0.1513 \\
$x_{2}$ & 0.1375 & 0.1409 & 0.1445 & 0.1478 & 3222 & 3403 \\
$x_{3}$ & 2500 & 2681 & 2861 & 3042 & 74 & 74 \\
exit & 149 & 102 & 73 & 73 & 3403 \\
\hline
\end{tabular}

As follows from the data in the table, starting with the third option, a subsequent increase in factors does not lead to any changes in the yield, that is, to an increase or decrease in the microbiological contamination of the water sample.

Analysis of the output of each of the options allows us to draw conclusions about the optimal ratios of factors for disinfecting a water sample: operating voltage of $19.9 \mathrm{kV}$, capacitance of $0.1445 \mu \mathrm{F}$ and the number of discharges 2861 pieces.

\section{CONCLUSION}

A complete factorial experiment was carried out. Thus, the influence of design parameters and exposure modes of an electro-hydraulic installation on the properties of water during the generation of highvoltage spark discharges is investigated. The design parameters were the additional spark gap and working gaps. Variable factors, that is, exposure modes, were taken as the operating voltage, capacitance of the capacitors and the number of electrical impulse discharges. Experimental data have been obtained on measuring the level of microbiological contamination of a water sample, which, according to an analysis of the data obtained, is reduced, which can serve as the basis for the possibility of the potential use of highvoltage discharges as a method of preparing water and its solutions under irrigation in greenhouses. The optimal ratios of factors for disinfecting a pond water sample from a source of artificial origin were identified: operating voltage $19.9 \mathrm{kV}$, capacitance $0.1445 \mu \mathrm{F}$ and the number of discharges 2861 pieces.

\section{REFERENCES}

[1] Kholmanskiy A., Smirnov A., Sokolov A., Proshkin Y., "Modeling of extraction mechanism of mineral elements by plants", Current Plant Biology, pp. 100-104, 2019.

[2] McGehee C.S., Raudales R.E., McAvoy R.J., Elmer W.H., "Efficacy of biofungicides against root rot and dampingoff of microgreens caused by pythium SPP", Crop Protection, vol. 121, pp. 96-102, 2019.

[3] Artamonov A.V., Izmailov A.Yu., Kozhevnikov Yu.A., Kostyakova Yu.Yu., Lobachevsky Ya.P., Pashkin S.V., Marchenko O.S., "Effective purification of concentrated organic wastewater from agro-industrial enterprises, problems and methods of solution", AMA, Agricultural Mechanization in Asia, Africa and Latin America, vol. 49, No. 4, pp. 49-53, 2018

[4] Echevarria C., Valderrama C., Cortina J.L., Martin I., Arnaldos M., Bernat X., De la Cal A., Boleda M.R., Vega A., Teuler A., Castellvi E., "Techno-economic evaluation and comparison of PAC-MBR and ozonation-UV revamping for organic micro-pollutants removal from urban reclaimed wastewater", Science of the total environment, vol. 671, pp. 288-298, Jun 2019.

[5] Lubello C., Gori R., Nicese F.P., Ferrini F., "Municipal-treated wastewater reuse for plant nurseries irrigation", Water Research, vol. 38, No. 12, pp. 2939-2947, 2004.

[6] Younis B.A., Mahoney L., Schweigkofler W., Suslow K., "Inactivation of plant pathogens in irrigation water runoff using a novel uv disinfection system", European Journal of Plant Pathology, vol. 153, No. 3, pp. 907-914, 2019.

[7] Benami M., Gillor O., Gross A., "The question of pathogen quantification in disinfected graywater", Science of the total environment, vol. 506, pp. 496-504, Feb 2015. 
[8] De la Hoz F., Rivera D., Arumí J.L., Chávez F., "Towards in-channel irrigation water disinfection using solar photocatalysis", Applied Engineering in Agriculture, vol. 25, No. 5, pp. 685-692, 2009.

[9] Lee S.J., Ma SH., Hong Y.C., Choi M.C., "Effects of pulsed and continuous wave discharges of underwater plasma on Escherichia coli", Separation and Purification Technology, vol. 193, pp. 351-357, Mar 2018.

[10] Zheng JS., "Inactivation of Staphylococcus aureus in water by pulsed spark discharge", Scientific Reports, vol. 7, art. numb. 10311, Sep 2017.

[11] Mudarisov S.G., Gabitov I.I., Lobachevsky Y.P., Mazitov N.K., Rakhimov R.S., Khamaletdinov R.R., Rakhimov I.R., Farkhutdinov I.M., Mukhametdinov A.M., Gareev R.T., "Modeling the technological process of tillage", Soil \& Tillage Research, vol. 190, pp. 70-77, 2019.

[12] Lenin K., Ravindhranath Reddy B., Surya Kalavathi M., "Blackfish optimization algorithm for solving reactive power problem", Indonesian Journal of Electrical Engineering and Informatics, vol. 4, No. 3, pp. 165-169, Sep 2016.

[13] Belov A.A., Vasilyev A.N., Musenko A.A., "Electrical conductivity of water treated by spark discharge", Indonesian Journal of Electrical Engineering and Informatics, vol. 7, No. 3, pp. 422-431, Sep 2019.

[14] Korotkov S.V., Andrianova M.Yu., Kozlov A.K., Korotkov D.A., Spichkin G.L., "A device for electrical-discharge treatment of water for biological investigations", Instruments and experimental techniques, vol. 62, issue 4, pp. 562565 , Oct 2019.

[15] Liu S.W., Liu Y., Ren Y.J., Lin F.C., Liu Ya., "Characteristic analysis of plasma channel and shock wave in electrohydraulic pulsed discharge", Physics of plasmas, vol. 26, issue 9, Sep 2019.

[16] Budnikov D., Vasilev A.N., "The model of optimization of grain drying with use of eletroactivated air", Advances in Intelligent Systems and Computing, vol. 866, pp. 139-145, 2019.

\section{BIOGRAPHY OF AUTHORS}

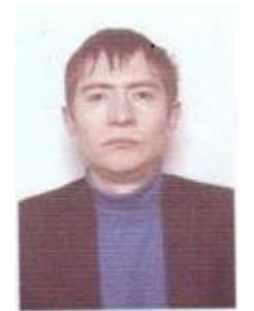

Alexander Anatolievich Belov - Dr.Sci. (Engineering), e-mail: belalexan85@ gmail.com Federal State Budgetary Scientific Institution «Federal Scientific Agroengineering Center VIM», Moscow, Russia.

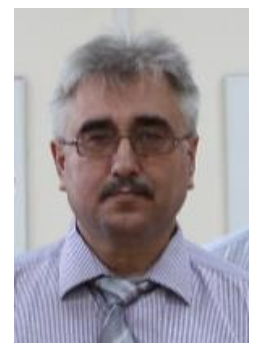

Alexey Nikolayevich Vasilyev - Dr.Sci. (Engineering), e-mail: vasilev-viesh@inbox.ru Federal State Budgetary Scientific Institution «Federal Scientific Agroengineering Center VIM», Moscow, Russia 